\title{
Tailored management of advanced squamous non-small cell lung cancer (NSCLC)
}

\author{
Matteo Giaj Levra • Silvia Novello
}

Published online: 18 December 2012

(C) Springer Science+Business Media New York 2012

\begin{abstract}
Lung cancer is the leading cause of cancer-related death worldwide, and squamous cell carcinoma is the second most frequent histological subtype. The improvement registered in the last years in the treatment of non-small cell lung cancer is attributable in part to the concept of personalized therapy, and to the possibility to transfer biological discoveries into clinical practice. Major changes have mainly characterized the adenocarcinoma histology, with the detection of the EGFR mutation and the EML4-ALK translocation, targeted by specific tyrosine kinase inhibitors. Despite its interesting oncogenic profile, squamous carcinoma is not the most appropriate example of "druggable disease", as it is characterized by deplorable results, often with a huge toxicity profile and without any benefit in terms of overall survival. At the present time, cytotoxic drugs are still the only treatment options for patients with advanced squamous cell carcinoma. Recent advances in the molecular characterization of squamous carcinoma have identified mutations and alterations, and clinical trials are ongoing to target these changes.
\end{abstract}

Keywords Squamous cell carcinoma - Targeted therapies · Genetic alterations · Tailored treatment

\section{Introduction}

Lung cancer is the leading cause of cancer-related death worldwide, with 1.4 million of deaths estimated in 2010 [1]. Approximately $85 \%$ of the newly diagnosed lung

\footnotetext{
M. Giaj Levra $\cdot$ S. Novello $(\bowtie)$

Department of Oncology, University of Torino,

AOU San Luigi, Regione Gonzole 10,

10043 Orbassano, TO, Italy

e-mail: silvia.novello@unito.it

M. Giaj Levra

e-mail: matteo.giajlevra@unito.it
}

cancers are non-small cell lung cancer (NSCLC) and, among these, $30 \%$ are squamous cell carcinoma (SCC) [2]. The incidence of this histology has become less common over the last decades, as a consequence of changes in smoking habits and cigarette manufacture, but it is still responsible of 40,000 deaths annually in the United States [3].

Recent advances in NSCLC drug development have introduced histology as an important key factor in the therapeutic strategy, and the simply dichotomy of small cell lung cancer (SCLC) and NSCLC is already outdated. Moreover, the possibility of targeting the activating epidermal growth factor receptor (EGFR) mutations and the anaplastic lymphoma kinase (ALK) translocation has led to a marked change in the treatment of a subgroup of patients who are mainly diagnosed with adenocarcinoma, which is the most common type of lung cancer $[4,5]$. These two alterations are not present in SCC; consequently, the targeted agents against these genomic alterations are largely ineffective for these patients.

In the past years, the attempt to introduce multi-target tyrosine kinase inhibitors (TKIs) and vascular endothelial growth factor receptor (VEGFR) inhibitors in the treatment of patients with advanced NSCLC showed an increased toxicity without significant benefit (often with a detrimental effect) in patients with the squamous subtype [6-8].

Recently, The Cancer Genome Atlas (TCGA) Research Network conducted a comprehensive study of lung SCC, with the aims of characterizing the genomic and epigenomic landscape of this cancer and identifing potential therapeutic opportunities. An integrated analysis, based on DNA copy number, somatic exonic mutations, messenger RNA sequencing, mRNA expression and promoter methylation, was performed in 178 SCCs. A large number and variety of DNA alterations were described, with a mean of 360 exonic mutations, 323 altered copy number segments and 165 genomic rearrangements per tumor. This project 
demonstrated that SCC is characterized by a higher mutation rate compared with adenocarcinoma, with a mean somatic mutation rate of 8.1 mutations per megabase $(\mathrm{Mb})$ versus 3.5 mutations per $\mathrm{Mb}[9 \cdot \bullet, 10]$.

The most common alterations discovered in SCC involve the following genes: CDKN2A, PI3KA, PTEN, FGFR1, EGFR, PDGFRA, CCND1, DDR2, BRAF, ERBB2, FGFR2 (Table 1).

This article aims to discuss those genetic alterations that could be a potential target for new biological drugs in patients with SCC.

\section{The phosphoinositide 3-kinase pathway}

The phosphoinositide 3-kinase (PI3K-AKT) is a signal transduction pathway that is crucial for cell survival, metabolism, motility and angiogenesis. The PI3K gene is found in the 3q26 amplified area, and its mutation encodes the alpha catalytic subunit of PI3K (p110 $\alpha$ ) of one of these kinases. The mutation rate of the PI3K gene in SCC, reported by the TCGA Network, was $16 \%$, not being mutually exclusive to EGFR or KRAS $[9 \bullet \bullet, 11 \bullet, 12]$. Several PI3K inhibitors are under investigation in clinical trials.

GDC-0941 (Genetech) is an oral selective pan-inhibitor of PI3K that inhibits common mutant forms of the PI3K p $110 \alpha$ subunit. In two open-label Phase Ia studies (GDC4254g and GDC4255g), patients with advanced or metastatic solid tumors were treated with GDC-0941 as

Table 1 Frequency of selected genetic abnormalities in squamous cell carcinoma $[9 \bullet \bullet]$

\begin{tabular}{llr}
\hline Gene & Event Type & Frequency \\
\hline CDKN2A & Deletion/Mutation/Metilation & $72 \%$ \\
PI3KCA & Mutation & $16 \%$ \\
PTEN & Mutation/Deletion & $15 \%$ \\
FGFR1 & Amplification & $15 \%$ \\
EGFR & Amplification & $9 \%$ \\
PDGFRA & Amplification/Mutation & $9 \%$ \\
CCND1 & Amplification & $8 \%$ \\
DDR2 & Mutation & $4 \%$ \\
BRAF & Mutation & $4 \%$ \\
ERBB2 & Amplification & $4 \%$ \\
FGFR2 & Mutation & $3 \%$ \\
\hline
\end{tabular}

CDKN2 Cyclin-dependent kinase inhibitor 2A, PI3KCA phosphoinositide 3-kinase, PTEN phosphatase and tensin homolog, FGFRl fibroblast growth factor receptor 1, EGFR epidermal growth factor receptor, $P D G F R A$ platelet derived growth factor receptor A, CCND1 cyclin D1, $D D R 2$ discoid-domain-containing receptor 2, BRAF v-raf murine sarcoma viral oncogene homolog $\mathrm{B} 1, E R B B 2$ v-erb-b2 erythroblastic leukemia viral oncogene homolog $2, F G F R 2$ fibroblast growth factor receptor 2 single agent. The maximum tolerated dose (MTD) of the drug administered daily for 21 days within a 28day cycle is $330 \mathrm{mg}$, with no dose limited toxicities (DLT) observed. One DLT of grade 4 elevated troponin was reported in patients treated with GDC-0941 at the dose of $330 \mathrm{mg}$ on a continuous day schedule. The only grade $\geq 3$ adverse event related to the study drug was one grade 3 hypokaliemia, while the other grade 1 and 2 adverse events described were: pneumonitis, nausea, diarrhea, rash, hyperglycemia and fatigue, with no deaths related to the study drug $[13,14]$. Several Phase I and II clinical trials are still open for accrual (Table 2).

BKM120 (Novartis) is an oral pan-PI3K inhibitor active against wild type and mutant isoforms; a Phase I trial investigated the MTD and the safety of this molecule in patients with solid tumors. The MTD of the drug administered on a continuous daily schedule was defined as $100 \mathrm{mg} /$ day on the basis of DLTs of mood alteration, hyperglycemia, epigastralgia and rash [15]. Currently, other clinical Phase I and Phase II studies evaluating DLT and safety of BKM120 monotherapy or in association with other agents are recruiting patients (Table 2).

PX-866 (Oncothyreon) is a synthetic derivative of wortannin, a natural furanosteroid metabolite product, isolated from a strain of Penicillium Wortmannii. It is responsible for an irreversible inhibition of PI3K, and in vitro and in vivo studies also demonstrated downstream of p-AKT, pS6 and p-mTOR. Hong and colleagues published the results of a Phase I study of PX-866 in patients with advanced solid tumors: the most common toxicity observed was diarrhea, mainly tolerable and well manageable, and in contrast to other PI3K inhibitors, no significant episodes of hyperglycemia and skin toxicity were reported. The recommended dose for a Phase II study was defined at $8 \mathrm{mg}$ daily [16].

\section{The AKT1 protein}

The v-akt murine thymoma viral oncogene homolog (AKT1) gene encodes for a protein kinase B, which is involved in the PI3K signaling [17]. The mutation E17K in this gene increases the activation of the PI3K pathway: this mutation was found in less than $1 \%$ of lung SCC, while no cases are described in literature for adenocarcinoma $[9 \cdot \bullet, 18]$.

MK-2206 (Merck) is a potent, orally, allosteric inhibitor of AKT1, AKT2 and AKT3, demonstrating tumor activity at pre-clinic level. A Phase I study is evaluating the DLT, MTD and the tumor activity of MK-2206 in combination with gefitinib in patients with NSCLC who failed prior chemotherapy and EGFR-tyrosine kinase inhibitor (Table 2). 
Table 2 Ongoing clinical trials for each pathway

\begin{tabular}{|c|c|c|c|c|c|}
\hline Pathway & Molecule & Monotherapy or Combination & Drug associated & Phase & Patients selection \\
\hline \multirow[t]{11}{*}{ PI3K } & \multirow[t]{3}{*}{ GDC-0941 } & Combination & $\begin{array}{l}\text { CBDCA-Pacl; CBDCA-Pacl- } \\
\text { Bev; CDDP-Pem-Bev }\end{array}$ & $\mathrm{Ib}$ & No \\
\hline & & Combination & Erlotinib & I & No \\
\hline & & Combination & CBDCA-Pac; CBDCA-Pac-Bev & II & No \\
\hline & \multirow[t]{7}{*}{ BKM120 } & Combination & Gefitinib & $\mathrm{Ib}$ & Yes-EGFR positivity \\
\hline & & Combination & CBDCA-Pac & I & No \\
\hline & & Combination & CBDCA-Pem & I & Yes-Non squamous \\
\hline & & Combination & Everolimus & I & No \\
\hline & & Combination & Docetaxel or Pemetrexed & II & Yes-PIK3CA activating mutations \\
\hline & & Monotherapy & - & II & Yes-PIK3CA activating mutations \\
\hline & & Combination & Erlotinib & I-II & Yes-Previous sensitivity to Erlotinib \\
\hline & PX-866 & Combination & Docetaxel & I-II & No \\
\hline \multirow[t]{5}{*}{ AKT1 } & \multirow[t]{5}{*}{ MK-2206 } & Combination & Gefitinib & I & Yes-Previous sensitivity to Gefitinib \\
\hline & & Combination & Erlotinib & II & Yes-Previous sensitivity to Erlotinib \\
\hline & & Combination & Erlotinib; AZD6244 & II & No \\
\hline & & Combination & Paclitaxel & I & No \\
\hline & & Monotherapy & - & II & No \\
\hline PTEN & GSK2636771 & Monotherapy & - & I & PTEN deficiency \\
\hline IGFR & AXL1717 & Monotherapy & Comparison versus Docetaxel & II & No \\
\hline \multirow[t]{3}{*}{ DDR } & \multirow[t]{3}{*}{ Dasatinib } & Monotherapy & - & II & No \\
\hline & & Monotherapy & - & II & $\mathrm{SCC}$ \\
\hline & & Monotherapy & - & II & DDR2 or BRAF mutation \\
\hline \multirow[t]{5}{*}{ FGFR } & BIBF 1120 & Combination & CDDP-Gem & I-II & $\mathrm{SCC}$ \\
\hline & AZD4547 & Monotherapy & - & I & No \\
\hline & BGJ398 & Monotherapy & - & I & No \\
\hline & E-3810 & Monotherapy & - & I & No \\
\hline & TKI258 & Monotherapy & - & II & No \\
\hline BRAF & Dabrafenib & Monotherapy & - & II & BRAF positive NSCLC \\
\hline
\end{tabular}

CBDCA carboplatin, CDDP cisplatin, Pacl paclitaxel, Bev bevacizumab, Pem pemetrexed, Gem gemcitabine (http://www.clinicaltrials.org, accessed 18 November 2012)

At the MD Anderson Cancer Center, the biomarker integrated targeted therapy study (BATTLE-2 Program) is enrolling patients with NSCLC who already received a firstline therapy. This is a Phase II, biomarkers driven study, in which patients are randomized in the arm which includes MK-2206 in association with erlotinib, or in association with AZD6244 (a MAP-extracellular signal-regulated kinase inhibitor) (Table 2).

\section{The phosphatase and tensin homolog}

The tumor-suppressor gene PTEN encodes for the phosphatase and tensin homolog, which has a tumor suppressor role with the downregulation of the PI3K-AKT-mTOR pathway; the loss of PTEN activity leads to a hyperactivation of the pathway [19]. The ATLAS research network reported the mutation of PTEN in $15 \%$ of SCC [9••].

GSK2636771 (GSK) is currently tested in a Phase $\mathrm{I} / \mathrm{II}$ in patients with advance solid tumors carrying PTEN deficiency (Table 2). A Phase I study of pazopanib in association with everolimus is recruiting patients with loss of PTEN (Table 2).

\section{The insulin-like growth factor-I receptor}

The insulin-like growth factor receptor (IGFR) is a cellsurface receptor with an intracellular tyrosine kinase domain. Its pathway is important in embryonic development, growth, metabolism, and in different tumor types, the abnormal activation of this signaling is described in literature 
[20]. The activation of the kinase domain of IGF-I or IGF-II stimulates downstream signaling, including the PI3K/AKT/ mTOR pathway and the RAS/RAF/MAPK one, promoting cell proliferation and inhibition of apoptosis [21].

The overexpression of IGF1R is more common in SCC than in adenocarcinoma; however, genetic activation of this pathway has not been reported [22, 23].

Figitumumab (CP-751,871; Pfizer) is a fully human, IgG2 monoclonal antibody against IGF-1R; the combination of figitumumab with carboplatin and paclitaxel was investigated in a Phase II, randomized trial, in chemonaïve patients with advanced NSCLC. The objective response rate in patients treated in the experimental arm was higher than in the control arm (54\% versus $42 \%$ ), and promising activity was seen especially in SCC $[24,25]$. The accrual in the Phase III trial for patients with NSCLC (squamous, large cell and adenosquamous carcinoma histology) was permanently suspended for futility, based on a planned interim analysis reporting an increased risk of early death in the experimental arm [26].

Dalotuzumab (MK-0646; Merck) is a humanized IgG1 monoclonal antibody against IGF-1R that was investigated in a Phase I/II trial in previously treated patients with NSCLC. The most common adverse events were grade 1 or 2 gastrointestinal disorders, skin toxicities, metabolic and nutritional disorders [27].

The accrual of patients in a randomized Phase II study of MK-0646/Placebo in combination with gemcitabine and carboplatin is already closed, but final data are not yet available.

\section{The discoid domain receptor 2 kinase}

The discoid domain receptor (DDR) is a tyrosine kinase that interacts with collagen and regulates cell adhesion, proliferation and extracellular remodelling [28]. The upregulation of DDR1 has been associated with an improved disease-free and overall survival in early stage NSCLC [29]. Mutations of DDR2 have been described in lung cancer, and the TCGA reported a $4 \%$ rate in SCC [9••].

In vitro and in vivo studies established that DDR2 mutant cell lines are sensitive to RNA interference and to dasatinib, a tyrosine kinase inhibitor used in the treatment of chronic myelogenous leukemia. In a Phase I/II clinical trial combining erlotinib and dasatinib, one patient with a SCC and DDR2 mutation exhibited a response to treatment after 2 months of therapy; the treatment regimen was continued for 14 months and then discontinued secondary to toxicities [30•]. According to these preliminary data, three Phase II trials are ongoing for patients with advanced NSLC: in one trial, there is no patient population enrichment and all NSCLCs are eligible, while in the others there is a selection for those patients with SCC, or for those harboring the DDR2 or the inactivating BRAF mutation (Table 2).

Preliminary in vitro studies showed that imatinib and nilotinib are also able to inhibit DDR1 and DDR2; in cell lines, these drugs led to a clear reduction of DDR autophosphorylation, with a consequent significantly inhibition of DDR1 and DDR2 kinase activity [31, 32].

\section{The fibroblast growth factor receptor}

The fibroblast growth factor receptor (FGFR) is a transmembrane receptor tyrosine kinase that participates in the regulation of embryonal development, cell proliferation, differentiation and angiogenesis; the downstream signaling cascade, transferring the signal to the nucleus involves either the Ras/MAPK or the PI3/Akt pathway [33•, 34].

The FGFR family includes four members (FGFR 1-4), and tumor growth depends on FGFR1 activation; the amplification of this gene showed a correlation with cigarette smoking in a dose-dependent manner, and in resected lung SCC it represents a negative prognostic factor [35, 36]. The chromosomal region at $8 \mathrm{p} 12$ spanning the FGFR1 gene locus is amplified in up to $15 \%$ of patients $[9 \bullet \bullet$.

Nintendanib (BIBF 1120; Boehringer Ingelheim Pharmaceuticals) is an oral inhibitor of VEGF, FGF and platelet-derived growth factor receptors. In a Phase I study, the association of BIBF 1120 combined with carboplatin and paclitaxel in 26 advanced NSCLC chemonaïve patients, showed a MTD of $200 \mathrm{mg}$ twice a day, with a toxicity profile characterized by gastrointestinal disorders (nausea, diarrhea and vomiting) and elevation of serum AST and ALT levels. Twelve patients achieved a partial response and two of these had SCC and one patient had a mixed large cell/squamous cell histology [37].

A Phase I/II study is enrolling chemonaïve patients with SCC to receive cisplatin and gemcitabine with or without BIBF 1120 (Table 2).

Other inhibitors of FGFR1 are under investigation in preliminary Phase I-II studies, including: AZD4547 (Astra Zeneca), BGJ398 (Novartis), E-3810 (Advenchen) and TKI258 (Novartis) (Table 2).

\section{B-RAF}

The B-RAF protein is a serine/threonine kinase that links RAS GTPases to downstream proteins of the MAPK family, which control cell proliferation; somatic mutations of BRAF were originally identified in melanoma $[38,39]$. The mutations of B-RAF are associated with increased kinase activity, with a constitutional activation of MAPK2 and MAPK3, and they are mutually exclusive with EGFR and 
KRAS mutation [40]. Mutations are seen in around $4 \%$ of patients with SCC [9・•].

Dabrafenib (GSK2118436, Glaxo Smith Kline) is a potent ATP-competitive inhibitor of BRAF kinase, whose activity has been described in patients with B-RAF mutant melanoma [41, 42]. A Phase II, non-randomized, open label trial in patients with NSCLC and BRAF mutations is still recruiting patients (Table 2).

\section{The sex-determining region Y-Box 2}

The sex-determining region Y-Box 2 (SOX2) is a transcription factor that has an important role in embryogenesis, especially in squamous differentiation of the esophagus and lung [43]. SOX2 amplification and expression have been detected in preinvasive lesions of the lung, such as squamous dysplasia and carcinoma in situ [44]. The SOX2 gene is amplified in about $20 \%$ of SCC; some studies reported an improved prognosis in patients with higher expression and amplification of this gene $[9 \bullet \bullet, 45]$. No SOX2 inhibitors are currently tested in clinical trials.

\section{The epidermal growth factor receptor}

Data about the presence of the activating EGFR mutation in SCC are still debated and controversial: the range of prevalence for this histology reported in literature is $1-15 \%$ [46•]. The major confounding factor about these data is a misinterpretation of the histotype: poorly differentiated adenocarcinoma and squamous cell carcinoma can appear indistinguishable, mainly on small biopsies [47]. Another confounder is the nonaccurate diagnosis of the adenosquaomus carcinoma (representing $0.4-4 \%$ of NSCLC), which cannot be reliable when achieved in small tissue fragments. Adenosquamous carcinoma harbor a spectrum of EGFR and KRAS mutations that is similar to adenocarcinoma $[46 \bullet, 48]$.

Rekhtman and colleagues evaluated 95 resected SCC for several activating mutations and none of these cases was positive for EGFR mutation; this result was confirmed in the study conducted by the TCGA Network. However, amplifications of EGFR were found in $7 \%$ of cases, including the Leu861Gln EGFR mutations, which seems to confer sensitivity to erlotinib and gefitinib $[9 \cdot \bullet, 46 \bullet]$.

About $5 \%$ of SCC presents the type III EGFR deletion mutant (EGFR vIII), resulting from a genomic deletion of exons 2 to 7 , which causes a deletion in the extracellular domain, leading to a proliferative advantage in cells expressing these truncated receptors [23]. Preclinical data suggest that reversible EGFR inhibitors would not have significant activity against the vIII mutation [49].

\section{The poly (ADP-ribose) polymerase inhibitors}

Poly (ADP-ribose) polymerase (PARP) is a nuclear protein that is activated when DNA is damaged, and PARP-1 is one of the most extensively studied of all the PARP family proteins [50]. The action of PARP-1 is essential in the repair of single-strand DNA breaks, predominantly through the base excision repair (BER) mechanism.

Since PARP proteins act as DNA damage "sensors", that allow a modulation of BER capacity, PARP is a potential target for inhibition of DNA repair. Modulation of polyADP ribosylation is therefore considered as a promising approach in clinical practice [51].

However, little is known about the frequency and the impact of sporadic loss of PARP1 expression in NSCLC and the utility of PARP proteins as a biomarker in lung cancer remains to be established [52].

Iniparib (BSI-201, Sanofi Aventis) is a molecule with very weak PARP-inhibitory properties and its real mechanism of action is yet to be elucidated [53]. Promising results from a Phase Ib trial about the activity of iniparib $5.6 \mathrm{mg} / \mathrm{kg}$ in association with carboplatin and paclitaxel were presented in 2011, showing an increased overall response rate compared with previous studies with carboplatin and paclitaxel alone. Three patients had a confirmed partial response; nine patients showed a stable disease, with only a progressive disease. The most common toxicities were grade 1-2 asthenia, anemia and nausea; grade $3-4$ adverse events were exclusively neutropenia (13.3\%) and anemia (6.7\%) [54].

At the 2012 IASLC (International Association for the study of Lung Cancer) worldwide meeting, the preliminary results of a randomized, open label Phase II trial of gemcitabine in combination with cisplatin, with or without iniparib $5.6 \mathrm{mg} / \mathrm{kg}$ in patients with advanced NSCLC were presented. The overall response rate for the iniparib arm was $21 \%$ (with one complete response) and $26 \%$ for the control arm, and no differences in response by subgroups were seen. The preliminary results of progression free survival showed a trend without a statistical significance in favor of the experimental arm with iniparib, while the toxicity profile of the combination of cisplatin, gemcitabine and iniparib was similar to that reported in the standard arm, with asthenia, nausea and neutropenia as most common adverse events [55].

The data from these two studies and those from triple negative breast cancer showed a synergy between the chemotherapic agents gemcitabine and carboplatin and iniparib, with a good tolerance [56]. A platinum-based doublet represents the gold standard in the first-line treatment for squamous carcinoma: the ECLIPSE study, a Phase III trial for patients with advanced squamous cell lung cancer receiving a combination of carboplatin gemcitabine either with or without BSI-201, was conducted and has recently completed the accrual. 


\section{Conclusions}

The treatment of lung cancer has been revolutionized, switching from a "one size fits all" approach to a personalized treatment. This new concept of personalized treatment is more common and successful in adenocarcinoma with the discovery of EGFR mutations and EML4-ALK translocation, while, until now, for SCC no alternatives to standard chemotherapy are available in clinical practice for the front-line treatment.

The interest and specific research of molecular diagnostics in SCC are continuously progressing, and over the last years many genomic alterations have been described, thanks to the development of next-generation genomic analyses. Recent data published by the TCGA Network are the first steps and the basis for the study of SCC genomic alterations, and although most of these mutation are identifiable in a small portion of patients, their discovery and the development of appropriate target agents could lead to an improvement in overall survival for these patients.

Many molecules are nowadays under investigation in Phase I and Phase II clinical trials, mainly with robust and promising preclinical data. Unfortunately, until now, no single Phase III trial evaluating targeted therapies had identified some benefits in the population of SCC, while a huge toxicity profile was frequently described for these patients.

In order to improve our knowledge about SCC and new therapeutic approaches, it is important that patients harboring specific molecular abnormalities such as PIK3CA mutations, FGFR1 amplifications and DDR2 mutations are enrolled in clinical trials testing the efficacy of new drugs specifically against these targets in this population.

Disclosure No potential conflicts of interest relevant to this article were reported.

\section{References}

Papers of particular interest, published recently, have been highlighted as:

- Of importance

-• Of major importance

1. World Health Organization. Cancer, fact sheet no. 297 http:// www.who.int/mediacentre/factsheets/fs297/en (accessed September 2012).

2. Travis WD. Pathology of lung cancer. Clin Chest Med. 2011;32:669-92.

3. Siegel R, Naishadham D, Jemal A. Cancer statistics 2012. CA Cancer J Clin. 2012;62:10-29.

4. Mok TS, Wu YL, Thongprasert S, et al. Gefitinib or carboplatinpaclitxel in pulmonary adenocarcinoma. N Engl J Med. 2009;361 (10):947-57.
5. Soda M, Choi YL, Enomoto M, et al. Identification of the transforming EML4-ALK fusion gene in non-small-cell lung cancer. Nature. 2007;448(7153):561-6.

6. Johnson DH, Fehrenbacher L, Novotny WF, et al. Randomized phase II trial comparing bevacizumab plus carboplatin and paclitaxel with carboplatin and paclitaxel alone in previously untreated locally advanced or metastatic non-small-cell lung cancer. J Clin Oncol. 2004;22(11):2184-91.

7. Scagliotti GV, Novello S, von Pawel J, et al. Phase III study of carboplatin and paclitaxel alone or with Sorafenib in advanced non-small-cell lung cancer. J Clin Oncol. 2010;28(11):1835-42.

8. Scagliotti GV, Wynnychenko I, Park K, et al. International, randomized, placebo-controlled, double-blind phase III study of motesanib plus carboplatin/paclitaxel in patients with advanced nonsquamous non-small cell lung-cancer: MONET1. J Clin Oncol. 2012;30(23):2829-36.

9. •- The Cancer Genome Atlas Research Network. Comprehensive genomic characterization of squamous cell lung cancers. Nature. 2012;489(7417):519-25. This study described an integrated analysis of 178 SC, with the identification of multiple somatic alterations that appears as possible drivers of pathways important in the biology of lung cancer.

10. Kan Z, Jaiswal BS, Stinson J, et al. Diverse somatic mutation patterns and pathway alterations in human cancers. Nature. 2010;466:869-73.

11. • Courtney KD, Corcoran RB, Engelman JA. The PI3K pathway as drug target in human cancer. J Clin Oncol. 2010;28:1075-83. In this review, the authors describe the role of PI3Kin tumor development and maintenance. They compared different therapeutic options to inhibit the PI3K pathway and data about the inhibitors of this pathway.

12. Thomas RK, Baker AC, Debiasi RM, et al. PI3KCA gene amplification in Japanese non-small cell lung cancer. Lung Cancer. 2007;58(1):159-60.

13. Baird RD, Kristeleit RS, Sarker D et al. A phase I study evaluating the pharmacokinetics (PK) and pharmacodynamics (PD) of the oral pan-phosphoinositide-3 kinase (PI3K) inhibitor GDC-0941. J Clin Oncol. 2010;28(suppl 15; abstr 2613).

14. Von Hoff DD, Lo Russo P, Demetri GD et al. A phase I doseescalation study to evaluate GDC-0941, a pan-PI3K inhibitor, administered QD or BID in patients with advanced or metastatic solid tumors. J Clin Oncol. 2011;29(suppl; abstr 3052).

15. Bendell JC, Rodon J, Burris HA, et al. Phase I, dose-escalation study of BKM120, an oral pan-class I PI3K inhibitor, in patients with advanced solid tumors. J Clin Oncol. 2012;30(3):282-90.

16. Hong DS, Bowles DW, Falchook GS, et al. A multicenter phase I trial of PX-866, an oral irreversible phosphatidylinositol 3-kinase inhibitor, in patients with advanced solid tumors. Clin Cancer Res. 2012;18:4173-82.

17. Testa JR, Bellacosa A. AKT plays a central role in tumorgenesis. Proc Natl Acad Sci USA. 2001;98(20):10983-5.

18. Malanga D, Scrima M, De Marco C, et al. Activating E17K mutation in the gene encoding the protein kinase AKT1 in subset of squamous cell carcinoma of the lung. Cell Cycle. 2008;7:665-9.

19. Sansal I, Sellers WR. The biology and clinical relevance of the PTEN tumor suppressor pathway. J Clin Oncol. 2004;22 (14):2954-63.

20. Dziadziuszko R, Camidge DR, Hirsh FR. The insulin-like growth factor pathway in lung cancer. J Thorac Oncol. 2008;3:815-8.

21. Pollak MN, Schemhammer ES, Hankinson SE. Insulin-like growth factors and neoplasia. Nat Rev Cancer. 2004;4:505-18.

22. Cappuzzo F, Tallini G, Finocchiaro G, et al. Insulin-like growth factor receptor 1 (IGF1R) expression and survival in surgically resected nonsmall cell lung cancer (NSCLC) patients. Ann Oncol. 2010;21:562-7.

23. Heist RS, Sequist LV, Engelman JA. Genetic changes in squamous cell lung cancer: a review. J Thorac Oncol. 2012;7(5):924-33. 
24. Karp DD, Paz-Ares LG, Novello S, et al. Phase II study of the efficacy of the antiinsulin-like growth factor type 1 receptor antibody CP-751,871 in combination with paclitaxel and carboplatin in previously untreated, locally advanced, or metastatic non-small cell lung cancer. J Clin Oncol. 2009;27:2516-22.

25. Correction: retraction. J Clin Oncol. 2012;30:4179.

26. Jassem J, Langer CJ, Karp DD, et al. Randomized, open label, phase III trial of figitumumab in combination with carboplatin and paclitaxel versus paclitaxel and carboplatin in patients with nonsmall cell lung cancer. J Clin Oncol. 2010;28:7500s.

27. Moran T, Felip-Font E, Sanchez Torres JM, et al. An open label, randomized $\mathrm{phI} / \mathrm{II}$ a trial evaluating MK-0646, an anti-IGF-1R monoclonal antibody, in combination with erlotinib for patients with recurrent non-small cell lung cancer. 100th AACR Annual Meeting, Apr 18-22 2009; Abstract 5622.

28. Labrador JP, Azcoitia V, Tuckermann J, et al. The collagen receptor DDR2 regulates proliferation and its elimination leads to dwarfism. EMBO Rep. 2001;2:446-52.

29. Ford CE, Lau SK, Zhu CQ, et al. Expression and mutation analysis of the discoidin domain receptors 1 and 2 in non-small cell lung carcinoma. Br J Cancer. 2007;96:808-14.

30. - Hammerman PS, Sos ML, Ramos AH, et al. Mutations in the DDR2 kinase gene identify a novel therapeutic target in squamous cell lung cancer. Cancer Discov. 2011;1:78-89. In this paper, the authors report novel mutations of DDR2 in 290 SCC samples. They present also the results in vitro and in vivo of the use of dasatinib in DDR2 positive cells lines, identifying a rationale for the use of this drug in SCC with DDR2 mutation.

31. Day E, Waters B, Spiegel K, et al. Inhibition of collagen-induced discoidin domain receptor 1 and 2 activation by imatinib, nilotinib and dasatinib. Eur J Pharmacol. 2008;599(1-3):44-53.

32. Rix U, Hantschel O, Durnberger G, et al. Chemical proteomics profiles of the BCR-ABL inhibitors imatinib, nilotinib, dasatinib reveal novel kinase and nonkinase target. Blood. 2007;110(12):4055-63.

33. - Turner R, Grose R. Fibroblast growth factor signaling: from development to cancer. Nat Rev Cancer. 2010;10:116-29. This paper presents a complete analysis of the signalling of the fibroblast growth factor, with the description of the activating mutations and the potential therapeutic approaches against this target.

34. Powers CJ, McLeskey SW, Wellstein A, et al. Fibroblast growth factor, their receptors and signaling. Endocr Relat Cancer. 2000;7(3):165-97.

35. Weiss J, Sos ML, Seidel D, et al. Frequent and focal FGFR1 amplification associates with therapeutically tractable FGFR1 dependency in squamous cell lung cancer. Sci Transl Med. 2010;2(62):62ra93.

36. Cho BC. Fibroblast growth factor receptor 1 gene amplification is associated with poor survival and cigarette smoking in resected squamous cell lung cancer patients. J Thorac Oncol. 2012;7(11): S455.

37. Doebele RC, Conkling P, Traynor AM, et al. A phase I, open-label dose-escalation study of continuous treatment with BIBF 1120 in combination with paclitaxel and carboplatin as first-line treatment in patients with advanced non-small-cell lung cancer. Ann Oncol. 2012;23:2094-102.

38. Leicht DT, Balan V, Kaplun A, et al. Raf kinases: function, regulation and role in human cancer. Biochim Biophys Acta. 2007; 1773:1196-212.

39. Davies H, Bignell GR, Cox C, et al. Mutations of the BRAF gene in human cancer. Nature. 2002;417:949-54.

40. Brose MS, Volpe P, Feldman M, et al. BRAF and RAS mutations in human lung cancer and melanoma. Cancer Res. 2002;62 (23):6997-7000.
41. Laquerre S, Arnone M, Moss K, et al. A selective Raf kinase inhibitor induces cell death and tumor regression of human cancer cell lines encoding B-RafV600E mutation. Mol Cancer Ther. 2009;8:abstr B88.

42. Falchook GS, Long GV, Kurzrock R, et al. Dabrafenib in patients with melanoma, untreated brain metastases, and other solid tumours: a phase 1 dose-escalation trial. Lancet. 2012;379:1893901

43. Bass AJ, Watanabe $\mathrm{H}$, Mermel $\mathrm{CH}$, et al. SOX2 is an amplified lineage-survival oncogene in lung and esophageal squamous cell carcinomas. Nat Genet. 2009;41(11):1238-42.

44. Yuan P, Kadara H, Behrens C, et al. Sex determining region Y-Box 2 (SOX2) is a potential cell-lineage gene highly expressed in the pathogenesis of squamous cell carcinomas of the lung. PLoS One. 2010;5:e9112.

45. Wilbertz T, Wagner P, Peterson K, et al. SOX2 gene amplification and protein overexpression are associated with better outcome in squamous cell lung cancer. Mod Pathol. 2011;24:944-53.

46. - Rekhtman N, Paik PK, Arcila ME, et al. Claryfing the spectrum of driver oncogene mutations in biomarker-verified squamous carcinoma of lung: lack of EGFR/KRAS and presence of PIK3CA/AKT1 mutations. Clin Cancer Res. 2012;18(4):116776. This paper clarifies the controversy of the real incidence of EGFR and KRAS mutations in patients with SCC, suggesting that in pure pulmonary SCC the EGFR/KRAS mutations do not occur. Most of positive EGFR/KRAS SCC are indeed adenosquamous carcinoma not well diagnosed.

47. Travis WD, Rekhtman N, Riley GJ, et al. Pathologic diagnosis of advanced lung cancer based on small biopsies and cytology: a paradigm shift. J Thorac Oncol. 2010;5:411-4.

48. Rektman N, Ang DC, Sima CS, et al. Immunohistochemical algorithm for differentiation of lung adenocarcinoma and squamous cell carcinoma based on large series of wholetissue sections with validation in small specimens. Mod Pathol. 2011;24:1348-59.

49. Ji H, Zhao X, Yuza Y, et al. Epidermal growth factor receptor variant III mutation in lung tumorgenesis and sensitivity to tyrosine kinase inhibitors. Proc Natl Acad Sci USA. 2006;103:781722.

50. Rouleau M, Patel A, Hendzel MJ, et al. PARP inhibition: PARP1 and beyond. Nat Rev Cancer. 2010;10:293-301.

51. Miwa M, Masutani M. PolyADP-ribosylation and cancer. Cancer Sci. 2007;98(10):1528-35.

52. Shiraishi K, Kohno T, Tanai C, et al. Association of DNA repair gene polymorphisms with response to platinum-based doublet chemotherapy in patients with non-small-cell lung cancer. J Clin Oncol. 2010;28(33):4945-52.

53. Tutt A. Synthetic lethality: targeting DNA repair mechanisms in clinical trials. Proceeding of AACR 102nd Annual Meeting; 2011 Apr 02-06; Orlando (FL), USA.

54. Mita AC, Mita MM, Rocha $\mathrm{C}$ et al. A phase $\mathrm{Ib}$ trial of iniparib (BSI-201) in combination with carboplatin (C) and paclitaxel (P) in patients with non-small cell lung cancer. J Clin Oncol. 2011;29: (suppl; abstr 7570).

55. Besse B, Felip E, Barlesi F, et al. Results of a randomized phase 2 trial of gemcitabine/ cisplatin/ iniparib vs gemcitabine/cisplatin (GC) in patients with stave IV NSCLC. J Thorac Oncol. 2011;6 (6):S469.

56. O'Shaughnessy J, Osborne C, Pippen JE, et al. Iniparib plus chemotherapy in metastatic triple-negative breast cancer. N Engl J Med. 2011;364(3):205-14. 